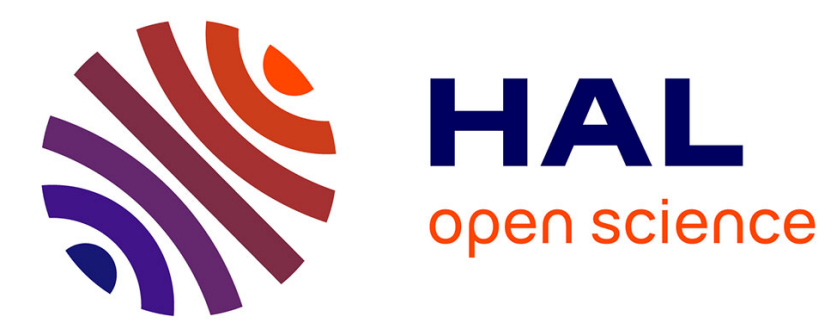

\title{
Critiques de trois arguments justifiant les lois sur le financement de la vie politique
}

\author{
François Facchini
}

\section{To cite this version:}

François Facchini. Critiques de trois arguments justifiant les lois sur le financement de la vie politique. Politiques et Management public, 2004, 22 (4), pp.27-46. 10.3406/pomap.2004.2854 . hal-00270469

\section{HAL Id: hal-00270469 \\ https://hal.science/hal-00270469}

Submitted on 4 Apr 2008

HAL is a multi-disciplinary open access archive for the deposit and dissemination of scientific research documents, whether they are published or not. The documents may come from teaching and research institutions in France or abroad, or from public or private research centers.
L'archive ouverte pluridisciplinaire HAL, est destinée au dépôt et à la diffusion de documents scientifiques de niveau recherche, publiés ou non, émanant des établissements d'enseignement et de recherche français ou étrangers, des laboratoires publics ou privés. 


\section{Critiques de trois arguments justifiant les lois sur le financement de la vie politique}

François Facchini ${ }^{1}$

Résumé : Cet article soutient que les lois sur le financement de la vie politique en France sont fondées sur de mauvais arguments. II soutient que la corruption ne s'explique pas par l'absence de lois sur le financement de la vie politique mais par la valeur des mandats(2), que les inégalités devant le succès électoral ne s'expliquent pas par le montant des dépenses de campagne mais par les résultats économiques des politiques publiques et le capital politique des candidats (3), et que l'action des groupes de pression ne remet pas en cause la sincérité du scrutin parce que le don ne doit pas simplement être pensé comme un acte d'investissement mais aussi comme un acte de consommation (4).

JEL : K00

Mots clés : corruption, capital politique, monnaie, politique et finance publique

\section{Introduction}

La thèse principale de la science juridique est que l'absence de réglementation du financement de la vie politique en France a provoqué des formes occultes de financement de la vie politique qui ont débouché sur les affaires et la mise en évidence d'une corruption généralisée au plus niveau de l'Etat. Les lois de 1988 sont alors naturellement expliquées par la lutte contre la corruption. A cet argument, les juristes ajoutent l'idée que l'argent décourage les candidatures et modifie les chances de succès des candidats en exerçant par la propagande qu'il permet une pression démesurée sur les électeurs ${ }^{2}$.

Cet article remet en cause ces trois arguments. II soutient sur la base des travaux de l'économie de la politique qu'il est peu vraisemblable que l'argent en politique ait pour conséquences de corrompre, de gagner les élections ou de rendre le scrutin moins sincère. La corruption n'est pas liée au processus de rivalité ou à l'absence de lois sur le financement de la vie politique, mais à la valeur des mandats (2). Le succès électoral n'est pas déterminé par le montant des dépenses de campagne, mais par les résultats économiques des politiques publiques et par le capital de notoriété des candidats (3). L'argent distribué par les groupes de pression lors des campagnes électorales n'achète pas les politiques publiques mais un accès à la vie politique (4).

\section{Moralisation de la vie politique et loi sur le financement de la vie politique}

Les lois de 1988, 1990, 1993 et 1995 ont pour principal objectif de lutter contre la corruption des mœurs politiques. Le législateur estime que la corruption est le résultat d'un vide juridique (Campana 1976) qui est d'autant plus dommageable qu'il s'est combiné à l'augmentation croissante du coût des campagnes électorales ${ }^{3}$ et à la mise en place des lois de décentralisation (Carsonne 1994, p.9). Nous pensons, au contraire qu'elle est fonction de la valeur du mandat.

II est facile, tout d'abord, de critiquer la thèse officielle qui soutient que l'augmentation des dépenses de campagne (Campana [1976], p.26, ou Uguen ([1995], Chapitre 1, Partie 2) a obligé les hommes politiques à trouver des financements occultes et illégaux ${ }^{4}$. La faible

\footnotetext{
${ }^{1}$ EDJ, Université de Reims Champagne Ardenne, Faculté de sciences économiques et de gestion, 57 bis rue Pierre Taittinger 51000 Reims, et Chercheur associé au LAEP, Université de Paris 1 Panthéon - Sorbonne, http://laep.univ-paris1.fr/facchini/index.htm

${ }^{2}$ Masclet (1984), mais aussi Pierre Joxe (1997, p.13).

${ }^{3}$ Aux Etats-Unis les dépenses de campagne électorale pour les élections présidentielles et pour les élections au sénat ont augmenté de 180\% en termes réels de 1976 à 1994 (Lott [2000], p.359).

${ }^{4}$ II est à juste titre rappelé "qu'un parti moderne (...)est une organisation structurée avec ses permanents, sa logistique, ses services d'étude, d'information, de relations publiques, et il doit faire
} 
rémunération publique des élus et l'augmentation des coûts de campagne sont alors interprétées comme les causes principales des comportements illégaux ou para-légaux ${ }^{5}$. Le raisonnement mène alors assez logiquement à l'intervention publique dans le financement de la vie politique. Si l'illégalité du financement de la vie politique s'explique par la faible rémunération des élus et l'augmentation inexorable des dépenses de campagne, alors il suffit d'augmenter la rémunération des élus ou de leur parti (rémunération indirecte) et de plafonner les dépenses de campagne électorale.

Cette explication de l'augmentation des budgets de campagne peut être jugée sommaire, comptable et immorale. Elle est sommaire parce qu'elle n'étudie pas l'évolution des dépenses de campagne électorale en longue période. Le taux de croissance des dépenses de campagne a par exemple suivi aux Etats-Unis l'augmentation du PIB, ce qui signifie qu'en moyenne le coût moyen de la démocratie rapporté au PIB est relativement stable ${ }^{6}$. Elle est comptable parce qu'elle n'explique pas pourquoi les candidats et les partis politiques utilisent des techniques de communication plus coûteuses. Elle est immorale, enfin, parce que ce n'est pas parce que le coût de la vie augmente que les consommateurs volent les biens dans les magasins. Ils acceptent de réduire leur niveau de vie. Ce n'est pas non plus parce que certains s'achètent des bateaux que ceux qui n'ont pas les moyens financiers de ce les acheter vont devenir des voleurs pour rendre leur demande de bateau solvable. II nous faut donc trouver une explication plus solide.

La nouvelle économie publique a proposé des explications plus sophistiquées sur les raisons de l'augmentation des budgets de campagne qui nous permettrons de lier de manière assez fine le problème de la moralisation de la vie politique et celui du financement de la vie politique.

\section{L'augmentation des dépenses de campagnes s'explique par l'augmentation de la valeur des mandats}

II est vraisemblable que l'augmentation des dépenses de campagne électorale s'explique par l'augmentation des dépenses publiques des gouvernements politiques. Le lien entre les deux variables (montant des dépenses de campagne électorale et montant des dépenses publiques) s'explique simplement par le fait que plus le gouvernement peut offrir de transferts publics plus les bénéfices des donateurs sont importants. II devient facile alors de comprendre que c'est parce que le gouvernement a plus à donner (régulation, subvention ou expropriation) que les donateurs sont prêts à dépenser plus et que les dépenses de campagne électorale augmentent inexorablement ${ }^{7}$ et cela quelles que soient les mesures législatives venant encadrer le financement de la vie politique. C'est parce que la valeur des

face à des tâches très lourdes. Il ne peut plus vivre d'expédients. Les problèmes de financement peuvent plus être résolus uniquement par les cotisations ni par les élus. Le parti doit accepter ou même solliciter l'aide des collectivités publiques, d'entreprises privées ou de groupements divers. II n'est pas besoin d'entrer dans le détail des procédés utilisés pour dire que beaucoup de ces recettes sont irrégulières et inavouables 》 (Masclet [1984], p.4).

${ }^{5}$ L'usage des fonds secrets est sans doute un exemple de pratiques para-légales.

${ }_{7}^{6}$ Remarque faite par Owens (1973) et citée par Amadany ([1977], p.295).

7 J. Lott ([2000], p.363) tente de falsifier son hypothèse ou de la vérifier par un travail économétrique sur les élections législatives et fédérales ainsi que sur la nomination des gouverneurs pour les EtatsUnis et conclut que son hypothèse est juste. L'augmentation des dépenses publics comme approximation de la capacité des gouvernements à créer des transferts explique l'augmentation des dépenses de campagne électorale. II constate, en effet, des différentiels significatifs entre les dépenses de campagne des différentes élections qui s'explique par des différentiels de dépenses publiques. Plus le poste à pouvoir donne de pouvoir budgétaire plus les dépenses de campagne électorale augmentent. II estime, par ailleurs, que les explications concurrentes proposées par la littérature comme l'altruisme, le nombre des candidats en compétition, ou la confusion des informations produite par les campagnes électorale sont moins solides d'un point de vue purement économétrique. 
mandats a augmenté que le montant des dépenses de campagne électorale a augmenté ${ }^{8}$. II existerait donc une relation entre le pouvoir législatif, réglementaire et budgétaire de l'élu et l'importance des fonds qu'il met en œuvre pour être réélu.

Le candidat est d'autant plus sollicité par les groupes d'intérêt et d'autant plus incité à obtenir le mandat que les bénéfices qu'il pourra en retirer sont importants ${ }^{9}$. L'augmentation du pouvoir de transfert entre les mains des gouvernements nationaux et locaux donne par conséquent une plus grande valeur aux fonctions politiques qui voient leur prix logiquement augmenter. Les individus qui contribuent à la victoire électorale du candidat sont prêts à dépenser plus d'argent parce qu'ils savent que le pouvoir de sanction et de récompense de l'élu est élevé. Les candidats qui s'engagent dans la compétition politique sont prêts à prendre les risque d'actions illégales parce qu'ils anticipent des opportunités de profits psychiques et monétaires importants.

\section{Augmentation des dépenses de campagne, valeur des mandats et corruption}

II devient alors tout naturel de lier l'augmentation des dépenses de campagne, l'augmentation des pouvoirs budgétaires des mandats politiques et la généralisation des comportements délictueux (Mises 1944, 1983), car il existe un lien étroit entre l'augmentation des pouvoirs budgétaires des mandats politiques et l'augmentation des comportements délictueux. C'est la trop grande quantité de règlement qui provoque l'apparition du clientélisme, du favoritisme et/ou de la corruption (Huntington 1968). Les occasions de corruption sont d'autant plus nombreuses que le nombre des personnes dépositaires de l'autorité publique s'accroît et que les prérogatives attachées à leur fonction, à leur mission et à leur mandat sont importantes. Le dépositaire de l'autorité publique est d'autant plus sollicité qu'il peut infliger des pertes ou accroître les profits des entrepreneurs. Les entrepreneurs vont alors chercher à obtenir la protection des hommes du gouvernement en finançant leurs campagnes électorales et leurs partis politiques (Mises 1944, p.77). C'est la bureaucratisation de l'économie qui crée donc des processus de recherche de profit inattendus et non désirés (Kirzner 1985, p.145).

De telles occasions n'existent que parce que les individus peuvent devenir des preneurs de règles lls peuvent grâce à leur action politique modifier les prix, la qualité et/ou les quantités. Les nouvelles règles du jeu qu'ils imposent aux autres joueurs induisent alors pour tout le monde de nouvelles occasions de pur profit mais aussi l'élimination d'autres occasions de profit qui en l'absence de changement dans les règles du jeu aurait existé (Kirzner 1985, p.144). Une forme d'occasion de profit naissant de l'intervention publique à laquelle on peut s'attendre est la corruption des hommes politiques et des administrations publiques. La corruption est une occasion de pur profit crée par l'intervention. Elle se présente comme un profit pur généré par l'intervention publique (Kirzner 1985, p.145). Elle conduit alors à une

\footnotetext{
8 Les lois de décentralisation ont favorisé la prévarication. Cette thèse est aussi celle des observateurs de la vie politique en France qui estiment que les lois de décentralisation de 1982-1983 ont favorisé une généralisation des comportements illégaux. II est accepté, en effet, que si «la décentralisation n'a pas créé le financement occulte qui lui préexistait, elle en a considérablement démultiplié et atomisé la population des responsables tentés d'y avoir recours, en même temps que des intermédiaires obligeant leur faisaient connaître les nombreuses techniques qu'ils auraient pu ignorer » (Carsonne [1994], p.10). Le contrôle d'une collectivité territoriale est un moyen de pratiquer la mise à disposition d'emplois fictifs, de voter des aides publiques aux groupes politiques et de mettre en place des systèmes de bureaux d'études effectuant des fausses factures (marchés publics, autorisation pour ouverture des grandes surfaces, dérogations, etc. Campana ([1976], p.123), Uguen ([1995], p.90-91). Voir aussi Uguen ([1955], p.76) et Bitoun P. (1993), La facture, Paris, Albin Michel expose l'importance des budgets de communication dans les budgets des régions métropolitaines.

${ }^{9}$ W. Crain et R. Tollison (1976) confirment l'hypothèse avancée par George Stigler selon laquelle les dépenses de campagne électorale des partis varient en fonction de la valeur marginale d'un élu pour le parti.
} 
dépravation de la vie publique et des affaires (Mises L. [1944], p.80, et Banfield E.C. 1975) qui accroît les coûts de la protection légale des droits de propriété et suscite alors logiquement une demande de plus en plus exigeante de la part des chercheurs de rente qui souhaitent que leur investissement en influence politique ait un rendement suffisant.

La corruption est, pour cette raison, l'un des facteurs les plus importants d'instabilité des économies mixtes, car si les coûts de protection légale (par la loi) des droits de propriété s'accroissent le citoyen peut prendre conscience qu'il a intérêt à protéger ses droits par les institutions judiciaires (par le droit). II tend alors progressivement à contester le système et à exiger un retour au droit qui limite considérablement le pouvoir budgétaire et la valeur des mandats pour les candidats. La moralisation de la vie politique devient, dans ces conditions, impossible si elle ne passe pas par une réforme de l'Etat et par un renforcement des limites constitutionnelles de l'action politique. "Si on veut réellement réduire les dépenses de campagne électorale, la solution est donc de réduire la puissance de l'Etat » (Lott ([2000], p.385). Le plafonnement des dépenses de campagne électorale agit sur les symptômes (augmentation des dépenses de campagne électorale), mais ignore les causes (Lott [2000], p.360). La liberté dans la recherche des moyens de financement n'est donc pas la cause de la corruption ${ }^{10}$ qui a des origines plus profondes.

\section{Rivalité politique, succès électoral et lois sur le financement de la vie politique}

Outre la thèse selon laquelle l'argent corrompt, le législateur soutient que l'argent biaise les résultats des élections en faveur des candidats qui dépensent le plus. L'économétrie du vote et la théorie du capital politique permettent, cependant, de préciser les conditions de validité de cette thèse.

\section{L'économétrie du vote et les déterminants du succès électoral}

L'économétrie du vote étudie de manière générale les déterminants du vote. Elle analyse plus particulièrement l'effet des dépenses de campagne sur le résultat des élections. Elle fait à cette occasion l'hypothèse que le vote des électeurs peut être traité comme un extrant d'une fonction de production dans laquelle la dépense de campagne apparaît comme un intrant. Elle cherche à connaître les effets des dépenses électorales sur les résultats des scrutins en construisant une fonction de production de vote (Lafay et Pommerehne [1983], p.4, François 2003). Ces résultats restent, cependant, difficiles à interpréter, d'une part, parce qu'une corrélation n'est pas une causalité et d'autre part parce que le montant des dépenses de campagne n'est pas le seul déterminant du vote. Toute augmentation des dépenses de campagne électorale du candidat rival diminuerait ceteris paribus les votes en faveur du candidat. Dans ces conditions, c'est plus le différentiel de dépense entre les candidats qui est important que le montant absolu de la dépense.

Les premières études en science politique ont montré statistiquement que les candidats qui gagnent les élections ont généralement dépensé plus que les perdants ${ }^{11}$. Pour les élections présidentielles de 1988 en France le candidat qui a dépensé le plus a été élu. L'analyse économique du vote a aussi mis en évidence des coefficients d'influence des dépenses de campagne électorale sur les résultats presque toujours statistiquement significatifs (Lafay et Pommerehne [1983], p.5). K.S. Palda (1973) montre pour les élections du parlement dans la province de Québec qu'il existe une relation positive entre les votes gagnés et les dépenses

\footnotetext{
${ }^{10}$ Michel Bouvier soutient cette position dans l'éditorial du numéro spécial de la revue française de finances publiques consacré au financement de la vie politique.

${ }^{11}$ David Adamany ([1977], p.294) cite l'étude de John R. Owens (1973), Trends in Campaign Spending in California, 1958-1970: Tests of Factors Influencing Costs, Princeton: Citizens' Research Foundation. II cite aussi l'étude de 1972 intitulée, Congressional Campaign Finance Monitoring Project, Washington: Common Cause, 1974, pp.1809.
} 
de campagne électorale du candidat (relation négative pour le rival). W.P. Welch (1974) montre pour les élections au Congrès américain ("sénat et house of representatives") que la part des votes des candidats républicains augmente ceteris paribus, aux élections du Sénat de $0,097 \%$ et de $0,065 \%$ aux élections de la "house of representative " si on augmente les dépenses de campagnes électorale de $1 \$$ pour 1000 habitants sur une moyenne de respectivement $208 \$$ et $103 \$$. (Lafay et Pommerehne [1983], p.5). K.S. Palda (1975) trouve une corrélation positive entre les dépenses de campagne électorale et le succès des candidats pour les élections canadiennes. Deux études d'économie du vote sur le cas français ajoute à cette liste non exhaustives des résultats significatifs.

- F. Palda et K.S. Palda (1998) trouve une corrélation positive pour les élections législatives françaises de 1993.

- A. François et M. Foucault (2002) soutiennent qu'ils existent une relation entre les différentiels de dépense et le succès aux élections ${ }^{12}$.

Toutes ces études ne permettent pas cependant à elles seules de soutenir que c'est parce qu'un candidat dépense plus d'argent qu'il gagne les élections.

II est, tout d'abord, possible d'inverser le lien de causalité. Si le montant des dons est fonction des probabilités de succès du candidat, le candidat qui reçoit le plus d'argent est celui qui est estimé le plus à même de gagner les élections. "Dans ce cas, il est impossible de savoir si les candidats ont gagné parce qu'ils ont dépensé plus " toute chose égale par ailleurs » ou parce que leur victoire était attendue » (Lafay et Pommerehne [1983], p.11). La relation entre le montant des dépenses et le succès exige une théorie des décisions des donateurs.

Il est, ensuite, important de rappeler que les modèles de prévision électorale introduisent des variables économiques à côté de la variable popularité ${ }^{13}$. Le montant des dépenses de campagne électorale peut être logiquement lié aux taux de popularité. L'information politique peut faire connaître un candidat et dans une certaine mesure le rendre populaire. Le montant des dépenses de campagne électorale peut, en revanche, plus difficilement influencer les résultats macro-économiques qui restent globalement indépendants. S'il est possible de penser un lien entre popularité et résultats macroéconomiques, il reste en revanche, plus difficile de lier dépense de campagne et déterminants économiques du vote. Le succès électoral ne peut pas, pour cette raison, être uniquement déterminé par le niveau de popularité et plus spécifiquement par les dépenses de campagne.

II n'est pas possible, pour ces raisons, de soutenir sans autres développements que le succès électoral est déterminé par le montant des dépenses de campagne. Le succès électoral est plutôt déterminé par les succès et les échecs économiques des candidats sortants et par le niveau de popularité des candidats.

\section{Les dépenses de campagne électorale et le niveau de popularité des candidats}

La théorie du capital politique explique les raisons de cette popularité. Le niveau de popularité mesurerait globalement la notoriété des candidats, autrement dit leur capacité à provoquer un vote routinier d'une partie des électeurs. II avantage les candidats parce qu'il leur donne le statut de point de référence pour les électeurs qui adoptent un comportement routinier qui leur permet de ne pas chercher d'informations additionnelles sur les préférences idéologiques des candidats (Lafay et Pommerehne ([1983], p.7). C'est cette possession d'un capital de notoriété qui explique le succès électoral et non le montant des dépenses de

\footnotetext{
${ }^{12}$ Pour une étude détaillée de la littérature et une étude originale sur le cas français lire la thèse d'Abel François (2003) LAEP.

${ }^{13}$ Dubois E. (2003), "Les modèles politico-économiques explicatifs du vote en France, 1976-2003 », Document de Travail LAEP, Université de Paris 1 présente l'ensemble de cette littérature et leurs résultats.
} 
campagne. Le capital de notoriété d'un candidat se constitue de plusieurs manières : être sortant, avoir un nom, ou appartenir à un parti puissant sont les principales raisons de ce capital.

a) Le candidat sortant est dans la position de la firme installée. II bénéficie d'une position dominante qui lui permet de dépenser moins sans pour autant hypothéquer ses chances de réélection ${ }^{14}$. II a accès aux médias, peut faire financer une partie de ces coûts de voyage par son administration et peut envoyer la plupart du temps des informations gratuitement aux électeurs ${ }^{15}$. Le sortant est donc le mieux placé pour collecter des fonds pour sa campagne (Adamany ([1977], p.294). II joue sa réélection sur son bilan, l'environnement économique (Jacobson 1980, et [1985], p.95) et sa notoriété ${ }^{16}$.

b) Les candidats ne sont pas égaux en capital politique parce qu'ils ne portent pas le même nom et n'appartiennent pas au même parti. Ce qui explique que contrairement à ce que soutenait Demsetz ${ }^{17}$ le capital politique est transmissible (Lott 1986, 1987). Les électeurs ont en effet tendance à soutenir les candidatures des familles (enfants voir Laband et Lentz [1985], cousins, parents, etc.) des sortants parce qu'ils sont assurés d'une forte proximité idéologique. L'image de marque se transmet principalement par le nom du sortant. Coats et Dalton (1992) montrent que le nom du sortant a affecté la concurrence politique des élections au parlement britannique durant tout le XIXème siècle (1852-1880). Ils doutent en revanche que le titre de noblesse ait un effet positif sur les chances de succès du candidat ${ }^{18}$. La notoriété se transmet aussi par les partis politiques. Le nom d'un candidat est associé à un parti. Les partis, capitalisent l'image de marque de leurs membres, leur bilan et leur charisme ${ }^{19}$ parce qu'ils ont une espérance de vie plus importante que les candidats. Ils se présentent, ainsi, comme des partis d'alternance crédibles ${ }^{20}$. Le capital politique des partis se présente alors comme une source de succès des candidats qui ont leur investiture. L'électeur peut, en effet, ne rien savoir sur un candidat et pourtant voter pour lui parce qu'il appartient à un parti (Ortiz 2000, p.758). Le parti joue ainsi comme un signal et un réducteur

\footnotetext{
${ }^{14}$ L'originalité de l'article de J.M. Netter (1983) est de confirmer la thèse selon laquelle la publicité est une barrière à l'entrée par l'étude des effets différentiels des dépenses des campagnes électorales des candidats sortants et des candidats potentiels. II montre que sur le marché politique la propagande peut-être considérée comme une barrière à l'entrée (Netter [1983], p.519).

15 Entre 1954 et 1970 93\% des députés du Congrès aux Etats-Unis étaient réélus (Lafay et Pommerehne [1983], p.7).

${ }^{16}$ Le sortant a aussi la possibilité de mobiliser un trésor de guerre pour dissuader les challengers de s'engager dans la concurrence électorale (Hersch et McDougall 1994). Les économistes américains appellent ces réserves financières les « war chest campaign » et les traitent comme des barrières à l'entrée. Hersch et McDougall ([1994], p.632) soutiennent que les "war chest campaign" altèrent la concurrence politique parce qu'elles modifient la perception des gains du challenger.

${ }^{17}$ La thèse de Demsetz (1982) était que les barrières naturelles à l'entrée (marque) ne provoquent pas de pertes d'efficacité parce que les capitaux qui ont créé la barrière peuvent être mis aux enchères et transférés vers les producteurs les plus efficaces. Cette situation ne serait pas vérifiée sur le marché politique parce que Demsetz soutenait que l'image de marque était non transférable. Les dépenses de campagne électorale créeraient alors des barrières à l'entrée protégeant uniquement le sortant. Dés que ce dernier abandonne la politique, il détruit de manière irréversible et non cessible son capital de notoriété.

${ }^{18}$ On peut aussi se référer aux travaux de Yen S., Coats R.M. et Dalton T.R. (1992).

${ }^{19}$ Pour une analyse économique de la place des partis sur le marché politique voir Breton $A$. and Galeotti G. (1986), «A Economic Theory of Political Parties », Kyklos, vol.39, pp.47-62 ou Johns P. and Hudson J. (1998), "The role of Political Parties: An Analyse Based on Transitions Costs ", Public Choice, vol.94, 1-2, pp.175-189.

${ }^{20}$ L'économétrie du vote faite le plus souvent pour élections américaines confirme généralement que les candidats qui gagnent les élections sont le plus souvent les sortants . Voir : W.Robert Reed et Eric Schansberg (1992) constatent notamment que le taux de turnover des hommes politiques a constamment baissé de 1953 à 1991 pour les «federal legislatives offices ». II existerait donc de moins en moins de renouvellement dans la classe politique américaine.
} 
d'ignorance. II a un rôle cognitif important. L'argent collecté par le parti lui permet de remplir cette tâche. Les études françaises sur le personnel politique confirment cette explication du succès par l'héritage politique familial ou partisan (Patriat et Parodi 1992, Garraud 1988 cité par François 2003, p.300) $)^{21}$.

c) Les candidats n'ont pas non plus le même capital politique parce qu'ils n'ont pas la même notoriété avant d'entrer en politique (Bond, Covington et Fleisher 1985). De nombreux hommes politiques font de la politique parce qu'ils sont connus dans le monde du spectacle, du sport, des affaires, de la science, de l'armée, etc.. Le challenger peut, par conséquent, compenser son retard par une image de marque qui ne doit rien aux montants des dépenses de campagne électorale ou à son expérience politique (temps) (Coats et Dalton [1992], p.77).

d) Le cumul des mandats est pour conclure ce point un dernier moyen de se faire connaître sans dépenser d'argent et/ou d'accroître la productivité électorale des euros dépensés (François [2003], p.325). II réduit la concurrence politique et les risques de perte par une logique de diversification de portefeuille. II élargit le capital politique et accroît ainsi les chances de réélection.

La notoriété d'un candidat lui permet donc d'être populaire. Cette popularité explique qu'un euro dépensé par un candidat à fort capital politique n'a pas le même rendement électoral qu'un euro dépensé par un candidat à faible capital ${ }^{22}$. Ces différentiels de notoriété expliquent alors pourquoi un certain nombre d'économistes compare les dépenses de campagne à des dépenses publicitaires et soutiennent que l'argent en politique est à l'origine de barrières à l'entée ${ }^{23}$. La libre entrée ${ }^{24}$ sur le marché politique n'est pas seulement limitée par la loi (barrières institutionnelles) ${ }^{25}$, elle l'est aussi par les différentiels de notoriété ${ }^{26}$. Les dépenses de campagne sont des dépenses publicitaires. A ce titre, elles valorisent un capital qui lui préexiste. Elle ne crée pas un produit ou une image de rien ${ }^{27}$. Les dépenses électorales n'expliquent pas, par conséquent la popularité. Elles ne servent pas à gagner les élections, mais permettent de ne pas les perdre (maintenir sa position dominante).

${ }^{21}$ Parodi J.L. et Patriat C. (eds 1992), L'héridité en Politique, Paris, Economica, coll. Collectivités Territoriales. Garraud P. (1988) «La sélection du Personnel Politique Local », Revue Française de Science Politique, 38 (3) : 402-432, Juin.

${ }^{22}$ Un dollars dépensé par le challenger à la « House election » a une productivité marginale en termes de vote inférieure à un dollar dépensé par le sortant (Netter ([1983], p.515). J.R. Lott (1987) fait la même hypothèse et la même constatation. II explique cela par la constitution d'un capital politique, d'une notoriété.

${ }^{23}$ Cette thèse est inspirée par des auteurs comme Bain (1976), Comanor et Wilson (1974) ou Porter (1976) qui soutiennent que les firmes installées ont un avantage significatif sur les entrants potentiels et que pour un certain nombre de bien où la publicité est une méthode importante de promotion, la publicité peut créer des barrières à l'entrée (cité par [Netter 1983], p.510).

${ }_{25}^{24}$ G. Becker ([1958], p.106)

${ }^{25}$ Woghemuth (1999, p.182) rappelle un certain nombre de barrières institutionnels : la périodicité des élections, les règles pour être candidat, les règles d'entrée aux parlements, et la pluralité des systèmes de vote. II ajoute aux barrières institutionnelles et stratégie l'idée qu'il existerait des barrières innocentes (p.183) comme 1) le fait qu'il n'existe qu'un seul président de la république ou un nombre limité de siège à pourvoir, 2) l'idéologie, et 3) la réputation.

${ }^{26}$ Le gouvernement peut ériger des barrières constitutionnelles. II peut exister entre les parties opposées sur la scène politique une stratégie de coopération. II peut exister des stratégies de protection de la rente (Woghemuth 1999).

27 Des auteurs comme Bain (1976), Comanor et Wilson (1974) ou Porter (1976) soutiennent que les firmes installées ont un avantage significatif sur les entrants potentiels et que pour un certain nombre de bien où la publicité est une méthode importante de promotion, la publicité peut créer des barrières à l'entrée (cité par [Netter 1983], p.510). 
Cette conclusion permet de soutenir que la popularité ne s'acquiert que rarement par des dépenses électorales et que les barrières à l'entré $e^{28}$ sur le marché politique sont un effet des différentiels de notoriété et non des dépenses de campagne électorale.

\section{Limiter l'action des groupes d'intérêt sur la vie politique}

Le législateur estime pour conclure qu'une action réglementaire doit permettre d'empêcher les groupes de pression d'acheter des politiques publiques, autrement dit de biaiser la sincérité du scrutin. La loi estime qu'il faut neutraliser l'argent des groupes de pression, parce que le législateur pense que les donateurs de campagnes électorales sont en fait ceux qui dirigent le pays. Si le groupe qui finance le candidat attend en échange de son vote une mesure politique qui lui est favorable, il interfère avec la volonté de l'ensemble des électeurs. II rompt la relation de confiance qui unit les électeurs à ses représentants. L'homme politique n'est plus le représentant de tous les électeurs qui ont voté pour lui, mais le représentant des électeurs qui ont financé sa campagne électorale. L'argent corrompt non pas seulement les mœurs politiques. Elle corrompt aussi l'offre politique qui est dépendante des promesses faites aux donateurs (Campana [1976], p.13). La loi souhaite donc rétablir le principe de souveraineté des électeurs et de sincérité du scrutin (Masclet [1984], p.5).

Le législateur cherche à éviter que le pouvoir économique (les plus riches) capte le pouvoir politique pour conserver la structure institutionnelle qui sied à ses intérêts ${ }^{29}$. L'inégalité des richesses ne doit pas, par conséquent, annuler le principe un homme - une voix. C'est parce que l'argent est réparti inégalement qu'il est dangereux (Nichols (1974). En d'autres termes après avoir cherché à savoir si l'argent achetait les voix des électeurs il s'agit de savoir s'il achète les politiques publiques.

\section{Le positionnement du problème}

Les liens entre l'action des groupes de pression et les politiques publiques font l'objet d'une importante littérature à la frontière des sciences politiques et des sciences économiques. Après avoir été l'apanage de l'école marxiste ${ }^{30}$ qui étudiait les liens entre l'argent des patrons et les décisions politiques ${ }^{31}$, l'école des choix publics a renouvelé cette problématique par l'usage des catégories les plus élémentaires de la science économique à savoir les notions de consommation, d'investissement, d'employé et d'employeur ${ }^{32}$.

${ }^{28}$ G. Tullock (1965)
${ }^{29}$ Cela suppose que les individus votent uniquement en fonction de leur position dans l'échelle des
revenus.
${ }^{30}$ Bunel J. et Saglio J. (1979), L'action patronale, du CNPF au petit patron, PUF, Paris. Jeanneney 30 Bunel J. et Saglio J. (1979), L'action patronale, du CNPF au petit patron, PUF, Paris. Jeanneney
J.N. (1976), François De Wendel en République, l'argent et le pouvoir 1914-1940, Ed Seuil, Paris. Jeanneney J.N. (1983), L'argent caché. Milieux d'affaire et pouvoir politique dans la France du XX èmes siècle, Paris, Fayard. Lévy-Leboyer M. (Ed. (1979), Le patronat de la seconde industrialisation, éd. Ouvrières, Paris. Cette thèse est sans doute confortée par le fait que le CNPF a aidé quasi publiquement certains candidats (Jox 1997, p.12), "Le financement des partis politiques ", Revue Française de Finances Publiques, $\mathrm{n}^{\circ} 58$, pp.11-19.

${ }^{31}$ Raymond Aron conteste la pertinence de la thèse qui consiste à soutenir que les décisions politiques seraient dictées par les patrons des grandes entreprises industrielles françaises (Aron R. [1958], p.856) parce que "le personnel politique et les minorités dirigeantes de l'économie ne constituent pas une classe ayant conscience de son unité, de ses intérêts communs (...) La vision marxiste, celle d'une minorité économiquement possédante exerçant de ce fait tous les pouvoirs y compris le pouvoir politique, parait fort éloignée du réel ». La démocratie resterait caractérisée par la séparation et non la confusion des pouvoirs politique et économique. Le pouvoir politique n'est pas l'auxiliaire du pouvoir économique des entreprises parce que ce dernier est limité par la division des intérêts, l'existence de contre pouvoirs (syndicats) et l'opinion publique (Meynaud ([1958], p.944-945).

${ }^{32}$ Voir pour un survol de la literature Potters R. and Sloof R. (1996), "Interest Groups : A Survey of Empirical Models that Try to Assess their Influence ", European Journal of Political Economy, 12: $4^{\circ} 3$ 442. 
Avant toute chose il faut rappeler qu'un groupe d'intérêt qui fait pression sur les élus lors des campagnes électorales est une association d'électeurs. La question est de savoir ce qu'ils cherchent à faire lorsqu'ils engagent des ressources en politique. Plusieurs hypothèses peuvent être avancées.

- II est possible de soutenir que les groupes de pression cherchent à acheter une politique publique. Une fois élu le candidat sera lié par la promesse qu'il a faite aux représentants des groupes de pression et modifiera la structure des droits de propriété en leur faveur.

- $\quad$ Il est possible de soutenir que le groupe de pression cherche uniquement à acheter une participation à la vie politique. II souhaite que ces préoccupations soient pris en compte dans les débats publics afin d'influencer les électeurs et modifier le cours de la campagne électoral et le positionnement des candidats et de renverser ainsi l'opinion en leur faveur. II ne s'agit pas d'acheter une politique mais d'imposer leur manière de voir l'avenir d'un secteur en pesant sur le processus de production et de diffusion d'information à l'œuvre lors de toute campagne électorale.

- II est possible de supposer que le groupe de pression cherche à acheter le travail des candidats et de leur parti lors de la campagne électorale. Le groupe de pression achète à travers la campagne électorale une tribune. II utilise le travail des candidats comme un intrant dans sa fonction de production et de diffusion d'informations sur leurs opinions politiques. A cette hypothèse s'en ajoute une autre. Le groupe de pression peut très bien acheter une promesse de politique publique. Le candidat s'engage une fois élu à modifier la structure des droits de propriété. Dans ce cas, le donateur cherche à limiter les comportements opportunistes de l'élu qui une fois en poste peut renoncer à tenir ses promesses. On peut, alors, penser que son parti qui a toujours besoin d'argent pour agir rappellera à l'élu ses promesses d'hier. Le parti politique est conçu, dans cette perspective, comme un intermédiaire (le superviseur d'une relation principal-agent) qui a pour mission de rappeler aux élus leurs engagements. L'électeur est l'employeur, le parti un contrôleur (contre-maître) et l'homme politique un employé ${ }^{33}$.

On peut aussi penser l'action politique des groupes de pression comme un acte risqué. Dans cette perspective elle est un investissement et/ou un moyen de s'assurer,

- Si l'action politique des groupes de pression est un investissement comme pour toutes les dépenses d'investissement il faut calculer un taux de retour sur investissement. L'électeur dépense $x$ euros en vue d'en toucher $x$ plus un intérêt $y, y$ étant le gain espéré de la dépense. Cet acte d'investissement a les mêmes ambitions que sur le marché économique. II s'agit d'accroître ses bénéfices futurs en mobilisant lors des périodes électorales une partie de ses ressources. Le candidat et le parti qui soutient sa campagne peuvent prendre alors l'allure d'une firme dans lequel on peut investir des parts qui selon son efficacité conduira à un gain et/ou à une perte. La notion d'investissement introduit un risque qui rend le gain aléatoire. L'investisseur donne en fonction des profits qu'il anticipe si un candidat gagne les élections. Le montant des dons dépend de la valeur du mandat. Chaque don est calculé en fonction des bénéfices et des coûts du succès de chaque candidat et de ses probabilités de succès.

- L'aléa lié aux actions politiques des candidats une fois élu peut alors conduire à une autre hypothèse. Les groupes de pression achètent une assurance contre un risque de perte. II ne s'agit pas d'acheter une politique publique, mais de se couvrir contre le risque de voir se mettre en place une structure des droits de propriété qui serait

\footnotetext{
33 Voir l'article de Samuel Issacharoff and Daniel R. Ortiz (1999), "Governing Through Intermediairies", 85, Va. Law Review, 1627 pour une présentation de la littérature sur les parties comme intermédiaire et les critiques que l'on peut lui adresser. Le parti est un super-agent.
} 
défavorable au groupe. Le groupe de pression engage de l'argent à partir de deux critères: les chances de succès d'un candidat et la probabilité que ce candidat propose une politique publique qui ne soit pas bénéfique pour le groupe. Cela signifie que le groupe de pression qui n'attend ni bénéfice ni coût d'une action politique n'engage aucune ressource en politique. Pour qu'il s'assure il faut qu'il y ait la conjonction de deux risques; le risque qu'il soit élu et qu'il fasse une mauvaise politique. Le montant des contributions est donc une fonction croissante à taux décroissant des probabilités subjectives de succès des candidats et des anticipations de profits induits par le succès d'un candidat (ou l'échec d'un autre) ${ }^{34}$. II est normal, dans cette mesure, que les dons aient tendance à se concentrer sur les candidats dont les chances de succès sont les plus élevés et qui promettent le plus (Lott $2000)^{35}$. Cette proposition précise l'argument selon lequel ce n'est pas l'argent qui fait le succès mais les probabilités de succès (et la valeur du mandat) qui attirent l'argent. Les inégalités des chances de succès aux élections sont la cause et non la conséquence du montant des dépenses de campagne électorale. Cela signifie que si l'augmentation des chances de succès d'un candidat accroît le montant des dépenses de campagne, l'inverse n'est pas vrai.

Si on lie l'idée que le succès électoral est lié à la forte notoriété et la théorie de l'assuré il est alors possible d'affirmer que les groupes de pression financent en priorité les candidats à forte notoriété puis diversifient leurs dons afin de limiter les conséquences de leurs erreurs éventuelles sur les chances de succès des candidats. Ces calculs politiques leurs permettent d'acheter une sorte «d'assurance politique» (Aranson et Hinich ([1979], p.440) qui les couvrent contre des sanctions éventuelles d'un candidat qui une fois élu punirait les groupes qui ne l'ont pas soutenus.

Chaque figure du groupe de pression conduit ainsi à préciser sous quelles conditions l'argent nuit à la sincérité du scrutin.

\section{L'argent des groupes de pression et la sincérité du scrutin}

1) II est juste, tout d'abord, d'affirmer que si le groupe de pression cherche à acheter un accès à la vie politique son argent ne modifie pas la sincérité du scrutin. II pourrait modifier le succès électoral, mais l'analyse menée à la section 3 a déjà écarté cette hypothèse. 2) II est correct, ensuite, de penser que si le groupe de pression achète le travail des candidats une fois élu il agit sur la sincérité du scrutin. 3) II est logique, enfin, d'affirmer qu'alors qu'un groupe de pression qui s'assure contre un risque n'a pas d'emprise sur les politiques publiques (accident), un groupe qui investit dans la politique cherche, au contraire, par son action à capitaliser une organisation pour lui donner de meilleures chances de financer ses investissements et de gagner les élections. L'action de l'investisseur a donc comme fin de modifier le scrutin (gagner les élections) et à termes d'en changer sa sincérité. Reprenons ces trois propositions afin d'en préciser les conditions de validité logique.

a) La première question consiste à savoir s'il faut concevoir l'argent des groupes de pression comme un moyen d'acheter une politique publique ou comme un moyen de participer à la vie politique. II est difficile d'affirmer qu'un groupe de pression achète une politique. Cela signifierait, en effet, que l'homme politique engagé dans une campagne électorale est un producteur de politique publique et que les principales caractéristiques de cette dernière peuvent être connues ex ante comme pour l'achat d'une voiture. Cette hypothèse signifierait qu'il n'y a pas d'aléa et que les promesses

\footnotetext{
${ }^{34}$ Le montant des dons aux candidats dépend aussi des valeurs des mandats. Le prix des mandats augmente avec le pouvoir budgétaire et réglementaire qu'il confère.

${ }^{35} \mathrm{On}$ explique ainsi pourquoi les candidats dont les programmes sont les plus interventionnistes sont ceux qui réussissent le mieux à collecter des fonds importants (Lott 2000).
} 
électorales sont toujours tenues. L'absence de mandats impératifs ainsi que l'incertitude qui entoure la période pendant laquelle sera mise en place la promesse électorale, qui peut à tout moment s'avérer inopportune à réaliser pour le groupe mais aussi pour l'élu, rendent cette hypothèse peu plausible. Il est peu probable que le groupe de pression achète une politique. II est plus probable, en revanche, qu'il achète une participation à la vie politique. S'il ne peut pas être sûr que le candidat qu'il finance gagnera les élections et fera ce qu'il a promis, il est certain en revanche qu'en finançant la campagne électorale d'un candidat ce dernier parlera de ces problèmes économiques et fera prendre conscience aux autres électeurs que le groupe a des revendications légitimes.

b) La deuxième question renvoie à la question de savoir si le groupe de pression peut utiliser le travail des candidats une fois élu comme un intrant de leur fonction de production. Cette proposition est douteuse pour au moins quatre raisons.

- II faut rappeler que les candidats reçoivent de l'argent de groupes de pression aux intérêts parfois divergents. Ils ne peuvent pas, pour cette raison, agir que dans un seul sens. Ils sont tenus, cependant, via l'action du parti politique qui leur rappelle leur engagement électoral de tenir compte des points de vue des financeurs dans la négociation des réformes. Là encore on retrouve l'idée que l'argent permet d'accéder à la vie politique sans pour autant garantir une décision politique puisque cette dernière suppose toujours une négociation. L'argent n'achète pas le travail des politiques. II achète uniquement leur écoute.

- II faut, ensuite, ne pas oublier la lâcheté des relations qui peuvent exister entre le parti et le candidat une fois élu. Le parti peut rappeler ses engagements à l'élu (ex candidat), mais il ne peut pas lui dicter sa conduite. Les liens entre le candidat et le parti sont plus stricts que ceux qui existent entre le parti et l'élu. L'élu tiendra compte de l'action des groupes de pression durant son mandat, de sa contrainte électorale et de la conjoncture. II est tenu par un certain nombre d'aléa dont il n'a pas conscience au moment où il reçoit les dons des groupes de pression.

- II faut ne pas sous-estimer l'engagement idéologique des candidats. La thèse selon laquelle l'action de l'homme politique pourrait être achetée par les groupes de pression suppose implicitement qu'elle s'offre au plus offrant. Si l'homme politique valorise le critère moral ${ }^{36}$ au détriment des critères électoraux et financiers ${ }^{37}$, il ne privilégiera ni l'opportunité de gagner les élections ni celle de gagner plus d'argent, il soutiendra les positions des groupes qui sont les plus proches de ses opinions et délaissera les calculs purement électoraux. Les groupes d'intérêt ont alors intérêt à financer un homme politique qui possède les mêmes préférences politiques qu'eux. L'argent des groupes de pression ne modifie donc pas la sincérité du scrutin puisque les hommes ne changent pas leurs opinions en fonction des offres de financement qui leurs sont offerts mais que les offres leurs sont faites parce que les hommes politiques estiment que les revendications des groupes de pression sont légitimes. C'est le vote qui dans ce cas détermine le montant de la contribution. Le fait que les hommes politiques agissent en accord avec les préférences politiques des groupes qui ont financé leur campagne ne remet pas en cause, pour cette raison, la sincérité du scrutin. II signifie simplement que les opinions politiques des groupes de pression

\footnotetext{
${ }^{36}$ S.G. Bronars et John R. Lott (1997) cite à l'appui de cette idée un certain nombre d'études empiriques sur le personnel politique des Etats-Unis d'Amérique. Lott J.R. and Reed W.R. (1989), "Shirking and Sorting in a Model of Finite-Lived Politicians", Public Choice, $n^{\circ} .61$, pp.75- et Coker D.C. and Crain W.M. (1994), "Legislative Committees as Loyalty-Generating Institutions", Public Choice, n०81, pp.195.

${ }^{37}$ L'élu peut décider d'une politique en fonction d'un critère morale, électorale ou financier. S'il valorise le critère électoral, il estime les coûts électoraux d'une décision (perte de voix) et les coûts du reniement. S'il privilégie le critère financier l'homme politique s'assure dans ce cas contre une défaite électorale. II ne demande pas au groupe d'intérêt de l'argent pour financer sa campagne électorale, mais un poste pour assurer son avenir.
} 
engagées dans la campagne électorale ont réussi à obtenir la majorité ${ }^{38}$, autrement dit que le candidat a été élu parce qu'il soutenait les arguments avancés par le groupe de pression.

- Une élection n'est jamais jouée et l'argent n'achète pas les voix. L'électeur reste toujours souverain. II peut donc sanctionner un candidat qui s'est ouvertement engagé à défendre un groupe d'intérêt particulier.

L'argent engagé dans la campagne électorale reste donc principalement un moyen de donner la parole aux hommes politiques qui représentent le mieux les intérêts des groupes. On peut aussi supposer que les donations viennent pour récompenser la décision de l'élu et non pour acheter sa décision. Comme les études développent une analyse simultanée du vote et du don, il est impossible de savoir si l'argent vient avant l'élection ou après.

c) La troisième question porte sur l'opposition entre acheter une assurance contre un risque et/ou investir dans la politique afin de valoriser ses ressources.

II est utile pour répondre à cette question de rappeler la faiblesse des investissements en influence politique au regard des gains obtenus. Ce constat conduit à un paradoxe (Tullock 1972); si l'argent est roi en politique pourquoi y en a-t-il si peu? Le montant des dépenses de recherche de rente des groupes de pression est effectivement très faible par rapport aux gains obtenus par les individus appartenant à ses groupes. Ils notent, par exemple, que les producteurs de blé contribuent à hauteur de 3,3 millions de $\$$ aux campagnes électorales des candidats et des partis et gagnent en retour 22,1 milliards de \$ (Ansolabehere et al. 2002, p.11). Vu sous cet angle l'investissement politique a un taux de retour astronomique qui oblige à se demander pourquoi la concurrence entre les chercheurs de rente ne conduit pas à une augmentation exponentielle des contributions politiques (investissement).

Les raisons de ce paradoxe ne peuvent pas se trouver uniquement dans la limitation des dépenses de campagne ou dans la situation de monopole (Helpman E. and Persson T. 2001) des donateurs. Elles se trouvent vraisemblablement dans le fait que les donateurs ne raisonnent pas comme des investisseurs. Ils se moquent du retour sur investissement parce que leur don correspond plus à un acte de consommation du bien politique qu'à un acte d'investissement (Ansolabehere et al. 2002). Le donateur consommateur cherche uniquement à accéder à la vie politique et non à acheter une politique. Les dons sont conçus comme un moyen d'améliorer ses chances de voir un élu et/ou un candidat s'intéresser à la vie de son groupe et aux problèmes qu'ils rencontrent. Le don politique ne garantit pas d'avoir une influence sur les politiques publiques, mais seulement l'opportunité de fournir une information qui peut influencer le législateur (Ansolabehere et al. 2002, p.32). L'argent des groupes de pression n'achète pas, dans ces conditions, une politique publique, mais un accès à la négociation qui sera immanquablement engagée pour modifier la structure des droits de propriété lors des réformes que les candidats une fois élus devront mettre en œuvre pour répondre aux attentes des électeurs et des groupes d'électeurs. La faiblesse des dépenses en influence politique au regard des gains potentiels conduit donc à soutenir l'idée que les groupes de pression se conduisent plus en consommateur de bien politique qu'en investisseur.

Il est important pour conclure ce point de faire remarquer qu'il n'existe pas de liens très nets entre le montant des contributions et la politique publique. Le retour sur investissement en politique est incertain et ne remplace pas les logiques de réseaux d'influence qui caractérisent la sphère politique et sociale. L'argent ne semble pas, dans ces conditions, l'intrant le plus important pour modifier la sincérité du scrutin. Le groupe de pression a plutôt intérêt à agir directement auprès des élus et non durant la campagne électorale, car il pourra

\footnotetext{
${ }^{38}$ Nous n'ignorons pas cependant l'effet des groupes de pression lors du mandat. II s'agit cependant d'une autre dimension de la pression, car le groupe agit directement sur l'élu et sa décision.
} 
évaluer les conséquences de son action, acheter les décisions (pot de vin), etc. Le retour sur investissement est plus certain hors période électorale que durant la campagne (résultat incertain, réalisation des promesses incertaines).

L'ensemble de ces arguments converge donc vers la thèse selon laquelle l'argent des groupes de pression permet d'accéder à la politique et à ses réseaux d'influence, de s'assurer contre un risque politique et/ou de récompenser les élus et les partis qui ont été loyaux mais qu'elle n'achète pas les décisions publiques. L'argent des groupes de pression est partie prenante du processus de production et de diffusion des connaissances dont l'homme politique a besoin pour ajuster son offre politique à la demande. II est simplement pour les électeurs associés en groupe d'intérêt un moyen d'influencer l'opinion publique et ses représentants.

\section{CONCLUSION}

Les lois sur le financement de la vie politique en France seraient donc mal fondées si elles avaient pour ambition de moraliser la vie politique, de rendre les candidats égaux devant les électeurs et de lutter contre l'activité de lobbying des groupes de pression, car l'argent des campagnes électorales n'est ni la cause de la corruption, ni la cause du succès électoral, ni la cause des votes des représentants du peuple à l'assemblée. La corruption est plutôt liée à la valeur des mandats; le succès électoral au niveau de popularité (mesure du capital de notoriété) et aux résultats macroéconomiques des sortants et le manque de sincérité aux défaillances cognitives des institutions qui encadrent l'échange politique.

- a) II n'est pas juste, pour cette raison, de soutenir que les lois sur le financement de la vie politique vont moraliser la vie politique française, car la loi s'attaque aux symptômes (la corruption) et non à sa cause (la valeur du mandat).

- b) II n'est non plus correct de penser que la loi réussisse à rendre le marché plus contestable. Le capital de notoriété des candidats est une donnée de l'échange politique que le législateur a sous-estimé (volontairement ou non ?).

- c) II est enfin peu probable que la réglementation du financement de la vie politique rendre le processus de rivalité politique libre égale et sincère, car ce n'est pas l'argent qui corrompt les résultats des scrutins et rend les candidats inégaux ${ }^{39}$ mais plutôt la structure du marché.

Ces lois seraient donc fondées sur une vision erronée de la place de l'argent en politique. L'argent en politique sert à accéder à la vie politique, à s'assurer contre un risque politique, à contrôler l'opportunisme des élus (via l'action du parti), à financer les hommes politiques de leurs activités, à découvrir les préférences des électeurs, à persuader du bien fondé des propositions contenues dans les programmes politiques, à informer les électeurs et à soutenir la concurrence électorale. II est nécessaire et utile à la vie politique d'une démocratie représentative. II n'est pas juste, dans ces conditions, de laisser penser que l'augmentation des dépenses électorales durant les années avant la réglementation ne répondait à aucune nécessité politique et n'était qu'un simple gaspillage.

Outre la critique des fondements habituels des raisons qui fondent les lois sur le financement de la vie politique l'économie de la politique conduit ainsi à repenser la place de l'argent en politique et à réinterpréter la hausse spectaculaire des dépenses de campagne dans les grandes démocraties. Dans la perspective, proposée ici l'augmentation des dépenses et des dons s'explique par la hausse de la valeur des mandats et des revenus des électeurs et des groupes qu'ils constituent. Si l'argent achète une participation à la vie politique et que la vie politique est un bien de luxe il est juste, en effet, de soutenir que plus les électeurs sont

\footnotetext{
${ }^{39}$ Pierre Joxe ([1997], p.) affirme clairement de le numéro de la Revue Française de Finance Publique que François Mitterand ne croyait pas que le montant des budgets de campagne électorale permettait de gagner une élection.
} 
riches plus leurs dons en valeur absolue sont importants et/ou plus leurs dons relativement à leurs revenus augmentent. II est correct aussi d'arguer que la hausse des dépenses de campagne (meeting, affiches, tracts, programmes, etc.) vise à satisfaire le consommateur de politique qui souhaite en avoir pour son argent et pouvoir participer activement à la campagne électorale. Le don en politique est, en ce sens partiellement indépendant du résultat du scrutin. Cet article devra donc naturellement se prolonger par une étude plus approfondie du rôle de l'argent dans la coordination des actions humaines dans la sphère politique.

\section{Bibliographie :}

[1] Adamany D. (1977), "Money, Politics, and Democracy : a review essay », The American Political Sciences Review, 71, 289-304.

[2] Ansolabehere S., de Figueiredo J.M. and Snyder J. (2002), "Why is there so Little Money in US Politics ", NBER Paper Series 1050 Massachusetts Avenue, Cambridge, MA 02138 December.

[3] Alexander H.E. (1972), Money in Politics, Washington: Public Affairs Press.

[4] Aranson P.H. and Hinich M.J. (1979), "Some aspects of the political economy of election campaign contribution laws", Public Choice, 34, pp.435-461.

[5] Aron R. (1958), "Note sur le pouvoir économique ", Revue Economique, №6, nov., pp.849-858.

[6] Barron D. (1989), "Service-Induced Campaign Contributions and the Electoral Equilibrium", Quaterly Journal of Economics, February, pp.45-72.

[7] Becker G. (1958), "Competition and Democracy", Journal of Law and Economics, October, pp.105-109.

[8] Ben-Zion U. and Eytan Z. (1974), "On Money, Votes and Policy in Democratic Society", Public Choice, 17, pp.1-10.

[9] Bond J.R., Convington C. and Fleisher R. (1985), "Explaining Challenger Quality in Congressional Elections", Journal of Politics, May, pp.511-529.

[10] Bronards S.G. and Lott J. (1997), "Do Campaign Donations Alter how a Politician Votes?", Journal of Law and Economics, 317.

[11] Campana A. (1976), L'argent secret. Le financement des parties politiques et des campagnes électorales, Arthaud, Paris.

[12] Carsonne G. (1994), « Du non-droit au droit », Pouvoirs, n70, pp.7-17.

[13] Crain W. and Tollison R. (1976), "Campaign Expenditures and Poilitical Competition", Journal of Law and Economics, April, pp.177-188.

[14] Crain W., Deaton T. and Tollison R.D. (1977), "Legislators as taxi cabs: on the Valu of seats in the US House of Representation", Economic Inquiry, 15, pp.298-302.

[15] Coats R.M. and Dalton T.R. (1992), "Entry Barriers in politics and uncontested elections", Journal of Public Economics, 49, pp.75-90.

[16] Demsetz H. (1982), "Barriers to entry", American Economic Review, 72, pp.47-57.

[17] Endersby J.W. and Munger M.C. (1986), "The Impact of Legislator Attributes on Union PAC Campaign Contributions", Journal of Labor Research, 13, pp.79-97.

[18] Evans D. (1986), "PAC Contributions and Roll-Call Voting : Conditional Power », in Interest Group Politics, $2^{\text {nd }}$ ed., edited by Allan J. Cigler and B.A. Loomis, Washington, D.C.: Congressional Quaterly.

[19] Facchini F. (2000), "Les effets de l'absence de prix monétaire sur la coordination politique", Journal des économistes et des études humaines, vol.X, n²/3, juin/septembre.

[20] François (2003), Economie Politique des Ressources Affectées aux Campagnes Electorales: Analyse Théorique et Empirique, Thèse pour le Doctorat, Université de Paris 1 Panthéon-Sorbonne.

[21] Grier J. and Munger M. (1986), "The Impact of Legislator Attributes on Interest Group Campaign Contributions", Journal of Labor Research, 7, pp.347-361.

[22] Grier K.B. and Munger M.C. (1991), "Committee Assigments, Consituent Preferences, and Campaign Contributions", Economic Inquiry 29: 24-43. 
[23] Helpman E. and Persson T. (2001), "Lobbying and Legislative Bargaining", Advances in Economic Analysis and Policy 1, chapter 3.

[24] Hersh P.L. and McDougall G. (1994), "Campaign War Chest as a Barrier to Entry in Congressioonal Races", Economic Inquiry, vol.32, October, pp.630-641.

[25] Jacobson G. (1980), Money in Congressional Elections, New Haven: Yale University Press.

[26] Jacobson G. (1990), "The effects of campaign spending in House elections for old arguments", American Journal of Political Science, 34, 3, pp.334-362.

[27] Johnson L. L. (1985), "The Effectiveness of Savings and Loan Political Action Committees", Public Choice, 46: 289-304.

[28] Jones W.J. and Keiser R. (1987), "Issue Visibility and the Effects of PAC Money", Social Science Quaterly, 68:170-176.

[29] Kirzner (1978), "Government Regulation and the market discovery process", in his Peril of regulation: a market process approach, law and economics center occasional paper, University of Miami School of Law, Coral Gables, fl, section IV, pp.13-19 (repr. 1985) in his Discovery and the Capitalist Process, Chicago and London: University of Chicago Press.

[30] Laband D.N. and Lentz B.F. (1985), "Favorite sons: Intergenerational wealth transfers among politicians", Economic Inquiry, 23, pp.615-641.

[31] Lafay J.D. et Pommerhen W. (1983), Recherche économétrique sur les dépenses des campagnes électorales : un exposé, minéo.

[32] Lassale J.P. (1997), "Le financement de la vie politique aux Etats-Unis », Revue Française de Finances publiques, n`58, pp.57-68.

[33] Lott J.R. (2000), «A Simple Explanation for Why Campaign expenditure are increasing: the government is getting bigger ", Journal of Law and Economics, October, pp.359-393.

[34] Lott J.R. (1987), "Licensing and nontransferable rents", American Economic Review, 77, pp.453-455.

[35] Lott J.R. (1986), "Brand names and Barriers to entry in Political market", Public Choice, 51, pp.87-92.

[36] Masclet J.C. (1984), "Le prix de la démocratie. Recherche sur la réglementation des dépenses et des ressources des partis politiques et sur la question de leur financement public ", dans Mélanges en l'honneur de Paul Maris Gaudemet, Economica, Paris.

[37] Meynaud J. (1958), "Pouvoir politique et pouvoir économique », Revue Economique, N6, nov., pp.925-957.

[38] Nelson P. (1976), "Political Information, Journal of Law and Economics, August, pp.315-336.

[39] Netter J.M. (1983), "Political Competition and Advertising as a Barrier to Entry", Southern Economic Journal, 50, 2, October, pp.510-520.

[40] Nichols D. (1974), Financing Elections: The Politics of an American Ruling Class, New York, New York ViewPoints.

[41] Ortiz D.R. (2000), "Duopoly versus Autonomy: How the Two-Party System Harms the Major Parties", Columbia Law Review, vol.100: 753-774.

[42] Palda K. (1973), "Does Advertising Influence Votes: An Analysis of the 66 and 70 Quebec Elections", Canadian Journal of Political Science, Bd, 6.

[43] Palda K. (1975), "The Effect of Expenditure on Political Success", Journal of Law and Economics, December, pp.745-772.

[44] Palda F. and Palda K. (1998), "The Impact of Campaign Expenditures on Political Competition in the French Legislative Elections of 1993", Public Choice, vol.94, 1-2, pp.157154.

[45] Pittman R. (1977), "Market Structure and Campaign Contributions", Public Choice, 31, pp.37-52.

[46] Reed W.R. and Schanberg E. (1992), "The Behavior of Congressional Tenure over Time", Public Choice, 73, pp.183-.

[47] Tullock G. (1965), "Entry Barriers in Politics", American Economic Review, 55, pp.458466. 
[48] Tullock G. (1972), «The Purchase of Politicians », Western Economic Journal, 10: 354355.

[49] Ugen J.L. (1995), Les élus et l'argent, Pour débattre, Syros, Paris.

[50] Wohlgemuth M. (1999), "Entry Barriers in Politics, or Why Politics, Like Natural Monopoly, In Not Organised as an Ongoing Market-Process ", The Review of Austrian Economics, vol.12, n², November, pp.175-200.

[51] Yen S., Coats R.M. and Dalton T.R. (1992), "Brand Name Investment of Candidates and District Homogeneity: An Ordinal Response Model", Southern Economic Journal, 58, 4, April, pp.988-1002. 